\title{
Intergroup Rhabdomyosarcoma Group IIb
}

National Cancer Institute

\section{Source}

National Cancer Institute. Intergroup Rhabdomyosarcoma Group IIb. NCI Thesaurus.

Code C148017.

Regional disease with positive lymph nodes, excised. 